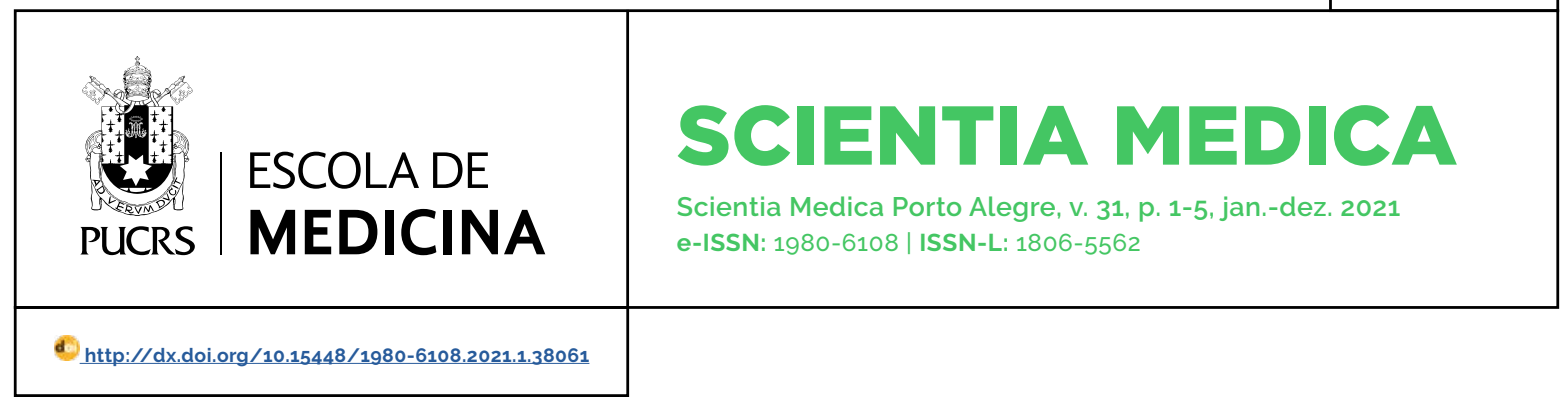

SEÇÃO: RELATO DE CASO

\title{
Benefícios do exercício físico na hipertensão arterial pulmonar associada à cardiopatia congênita não corrigida
}

\author{
Benefits of Physical exercise in pulmonary arterial hypertension associated with \\ uncorrected congenital heart disease
}

\section{Andrieli Barbieri Garlet ${ }^{1}$ orcid.org/0000-0003-2399-5867 andrygarlet@hotmail.com}

\section{Aline Tibola ${ }^{1}$ \\ orcid.org/0000-0001-9959-3069 aline-tb17@hotmail.com}

\section{Bruna Eibel ${ }^{2}$}

orcid.org/0000-0002-2077-3751 brunaeibel@gmail.com

Recebido em: 12 maio 2020 Aprovado em: 25 jan. 2021. Publicado em: 22 fev. 2021

\section{(c) (1)}

Artigo está licenciado sob forma de uma licença Creative Commons Atribuição 4.0 Internacional.

\section{Resumo}

Introdução: as cardiopatias congênitas são classificadas como um dos principais grupos para desenvolver hipertensão arterial pulmonar. O programa de exercicio físico pode auxiliar no aumento da capacidade de vida diária, melhora clínica e funcional, minimizando as consequências deletérias da patologia.

Relato do caso: reportamos um caso de um paciente do gênero masculino, 31 anos, com diagnóstico clínico de hipertensão arterial pulmonar grave em decorrência da dupla via de saída do ventrículo direito com comunicação interventricular, sem intervenção cirúrgica. Após ser encaminhado ao programa de Reabilitação Cardiopulmonar e Metabólica em um centro de referência em cardiologia do Rio Grande do Sul, foram realizadas avaliações clínicas e, também, da capacidade funcional pelo teste de caminhada de 6 minutos, da força muscular e resistência periférica, pelo teste Sentar-Levantar, e da força muscular respiratória, pelo exame de manovacuometria. Os valores apresentavam-se abaixo do valor previsto. O programa de exercício proposto foi realizado durante 30 sessões constando de exercicios aeróbicos com duração de 33 minutos, combinados ao treino de fortalecimento dos grandes grupos musculares.

Conclusão: após o término do programa observou-se um acréscimo de 83 metros no teste de caminhada de 6 minutos e um aumento da força muscular respiratória, assim como, redução redução nos parâmetros da escala de Borg.

Palavras-chave: exercício, cardiopatias congênitas, hipertensão pulmonar, reabilitação cardiaca, fisioterapia.

\section{Abstract}

Introduction: congenital heart disease is classified as one of the main groups to develop pulmonary arterial hypertension. The exercise program can help to improve daily living, capacity, clinical and functional improvement, minimizing the deleterious consequences of the disease.

Case report: we report a case of a 31-year-old male patient with a clinical diagnosis of severe pulmonary arterial hypertension due to the right ventricular double outflow tract with interventricular communication without surgical intervention. After being referred to the Cardiopulmonary and Metabolic Rehabilitation program at a cardiology reference center in Rio Grande do Sul, clinical evaluation was performed, as well as functional capacity by the 6-minute walk test, muscle strength and peripheral resistance by the sitting-rising test, and respiratory muscle strength by the manovacuometry exam. The values were below the predicted value. The proposed exercise program was performed during 30 sessions consisting of aerobic exercises lasting 33 minutes, added strength training of large muscle groups.

Conclusion: after the program there was an increase of 83 meters in the 6-minute walk test and an increase in respiratory muscle strength, as well as a reduction in Borg scale parameters.

Keywords: exercise, heart defects, congenital, pulmonary hypertension, cardiac rehabilitation, physiotherapy. 
ABREVIATURAS: CIV, comunicação interventricular; DVSVD, dupla via de saída do ventriculo direito; HAP, hipertensão arterial pulmonar; RCPM, Reabilitação Cardiopulmonar e Metabólica; SpO2, saturação periférica de oxigênio; T6', teste de caminhada de 6 minutos

\section{Introdução}

As cardiopatias congênitas são anomalias resultantes de defeitos anatômicos do coração ou de grandes vasos associados. A incidência brasileira corresponde a 1 em cada 1 mil nascidos vivos (1). Entre elas, a dupla via de saída do ventrículo direito é diagnosticada quando mais de $50 \%$ de cada artéria, pulmonar e aórtica, se origina do ventrículo morfologicamente direito e raramente não está associada a uma comunicação interventricular (2).

As cardiopatias congênitas são consideradas um dos principais grupos para desenvolver hipertensão arterial pulmonar (HAP), a qual é definida pela pressão arterial média pulmonar maior que $\geq 25 \mathrm{mmHg}$, pressão capilar pulmonar abaixo de $15 \mathrm{mmHg}$ e resistência vascular pulmonar > 3 unidades Wood (3).

A HAP é marcada pelo baixo volume de ejeção devido à sobrecarga imposta ao ventrículo direito. Isso corrobora com a reduzida capacidade de exercício, restrição ao consumo máximo de oxigênio e limiar anaeróbico, resultando em limitação da capacidade funcional. Além disso, há relatos de comprometimento da função muscular respiratória, com fraqueza e atrofia das fibras musculares diafragmáticas (4). Um programa de exercicio físico pode auxiliar no aumento da capacidade ao exercicio, melhora clínica e funcional, minimizando as consequências deletérias da patologia (5).

\section{Caso clínico}

Homem, 31 anos, raça branca, com diagnóstico clínico de dupla via de saida do ventrículo direito com comunicação interventricular subaortica com fluxo esquerda-direita, sem estenose pulmonar. $O$ sujeito estava apresentando HAP grave (pressão sistólica da artéria pulmonar $90 \mathrm{mmHg}$ ) associado à doença vascular pulmonar avançada, com fração de ejeção do ventrículo esquerdo de $68,77 \%$ e, fazia uso das seguintes medicações: sildenafil $60 \mathrm{mg} / \mathrm{dia}$, furosemida $40 \mathrm{mg} / \mathrm{dia}$, enalapril 20 $\mathrm{mg} / \mathrm{dia}$, carvedilol 12,5 mg/dia, espirolactona 25 mg/dia, digoxina 0,125 mg/dia, AAS 200 mg/dia.

A mal formação cardíaca foi diagnosticada pelo ecocardiograma fetal, com recusa do tratamento cirúrgico pelos pais. O paciente não realizou nenhuma intervenção medicamentosa ou cirúrgica prévia à reabilitação e relatou ser assintomático até os 22 anos, quando começou a apresentar dispneia e fadiga aos esforços moderados, então procurando atendimento médico. Foi optado pela não intervenção cirúrgica devido à resistência vascular pulmonar $>9$ Woods $8 / \mathrm{m}$ (2).

Com 31 anos de idade, foi encaminhado para - programa de reabilitação cardiopulmonar e metabólica em um centro de referência em cardiologia do Rio Grande do Sul. Na avaliação clinica, apresentava classe funcional NYHA II e como fatores de risco identificaram-se hipertensão arterial sistêmica, sedentarismo e estresse. Foram avaliados, também, capacidade funcional pelo teste de caminhada de 6 minutos (6), força muscular e resistência periférica pelo teste Sentar-Levantar e força muscular respiratória pelo exame de manovacuometria (7). O programa de reabilitação cardiopulmonar e metabólica constou de 30 sessões de exercício aeróbico com duração de 33 minutos. As avaliações encontram-se na Tabela 1.

TABELA 1 - Avaliação pré e pós-programa de reabilitação cardiopulmonar e metabólica

\begin{tabular}{lccccc}
\hline & Previsto & Pré & \% Predito & Pós & \% Predito \\
\hline T6', min [6] & 777 & 457 & 59 & 540 & 69.5 \\
PI máx, $\mathrm{cmH}_{2} \mathrm{O}[7]$ & -130 & -62 & 48 & -128 & 99 \\
$\mathbf{P E}$ máx, $\mathrm{cmH}_{2} \mathrm{O}[7]$ & 139 & 118 & 84 & 131 & 93 \\
$\mathrm{~S} / \mathrm{L}, \mathrm{n}^{\circ}$ repetições & $14-19$ & 15 & média & 19 & média \\
\hline
\end{tabular}

T6', teste de caminhada de 6 minutos; PI máx, pressão inspiratória máxima; PE máx, pressão expiratória máxima. S/L, sentar-levantar; Pré e Pós programa de reabilitação cardiopulmonar e metabólica. 
Durante o programa de exercícios utilizou-se oxigenioterapia de 4 a $5 \mathrm{~L} / \mathrm{min}$ para manter uma saturação periférica de oxigênio média de $80 \%$. Combinado ao treino aeróbico foram associados exercícios de fortalecimento dos grandes grupos musculares, com progressão da carga de exercício. O paciente iniciou o programa em bicicleta ergométrica e, após a décima sessão, evoluiu para esteira ergométrica. A evolução do programa de reabilitação é demonstrada na Tabela 2.

TABELA 2 - Evolução do programa de reabilitação cardiopulmonar e metabólica

\begin{tabular}{lccccccc}
\hline Sessão & $\mathbf{F C}_{\mathbf{i}}(\mathbf{b p m})$ & $\mathbf{F C}_{\mathbf{f}}(\mathbf{b p m})$ & $\mathbf{S p O}_{\mathbf{2 i}}(\%)$ & $\mathbf{S p O}_{\mathbf{2 f}}(\%)$ & Borg $_{i}$ & Borg $_{\mathbf{f}}$ & Distância (m) \\
\hline $\mathbf{1}^{\mathbf{a}}$ & 75 & 106 & 83 & 79 & 2 & 3 & $4600^{*}$ \\
$\mathbf{1 0}^{\mathbf{a}}$ & 66 & 77 & 87 & 89 & 1 & 2 & 1840 \\
$\mathbf{2 0}^{\mathbf{a}}$ & 74 & 60 & 92 & 93 & 0.5 & 1 & 2510 \\
$\mathbf{3 0}^{\mathbf{a}}$ & 78 & 90 & 86 & 85 & 0,5 & 1 & 2840 \\
\hline
\end{tabular}

Borg; percepção inicial fadiga/dispneia pela escala de Borg modificada; Borg f $^{\prime}$ Percepção final fadiga/dispneia pela escala de Borg modificada; FCi: frequência cardiaca inicial; FC $_{\text {f' }}$ frequência cardiaca final; SpO ${ }_{2 i}$ Saturação periférica inicial; $\mathrm{SpO}_{2 f}$ : Saturação periférica final;

*Distância percorrida na bicicleta ergométrica na $1 .^{a}$ sessão.

\section{Discussão}

A fisiopatologia da HAP está associada à redução na proporção de fibras musculares do tipo I, relação de fibras capilares, atividade enzimática aeróbica, prejuizo da biogênese mitocondrial e aumento da degradação proteica muscular (8), corroborando com a sensação de fadiga, angina e progressão da dispneia. Em seu estágio inicial o paciente pode ser assintomático (9).

A diretriz europeia de HAP recomenda como forma segura, juntamente com o tratamento medicamentoso, o treinamento físico e a reabilitação (grau II a, nivel B) (9). Além disso, o treinamento físico provoca adaptações que otimizam a cinética do oxigênio e da função cardiovascular. Individuos com fraqueza muscular respiratória podem ter uma capacidade funcional reduzida devido a um pico significativamente menor do consumo máximo de oxigênio (10).

Em um estudo que comparou individuos com HAP a outros individuos saudáveis, demonstrou que nessa patologia os sujeitos apresentaram significativa redução da capacidade funcional, no nivel de atividade física e na força muscular respiratória e periférica (11). Esses achados vão ao encontro do nosso estudo, que demonstrou eficácia no protocolo utilizado, principalmente em relação à força muscular respiratória, com aumento de 106\% na força muscular inspiratória pós-intervenção.

Uma meta-análise que avaliou a efetividade e a segurança do exercício físico em pacientes com HAP, constatou que ele parece seguro e está associado a uma melhora significativa do teste de caminhada de 6 minutos e da capacidade de exercício. Tal resultado é causado pelo aumento do consumo máximo de oxigênio e da redução da pressão arterial sistólica pulmonar de repouso (12). Nossos achados vão ao encontro da literatura e evidenciam que um programa de exercício aeróbico associado ao treinamento de fortalecimento muscular periférico, durante 30 sessões, promoveu uma melhora de $83 \mathrm{~m}$ percorrida no teste de caminhada de 6 minutos, em relação ao valor pré-intervenção.

Este relato de caso mostrou que o treinamento aeróbico associado ao treino de força é benéfico para a capacidade funcional, a força muscular ventilatória, a resistência muscular periférica e a sensação de dispneia, em um individuo com cardiopatia congênita não corrigida e HAP. 
Notas

\section{Apoio financeiro}

Este estudo não recebeu apoio financeiro de fontes externas.

\section{Declaração de conflito de interesses}

Os autores declaram não haver conflitos de interesses relevantes ao conteúdo deste estudo.

\section{Contribuições dos autores}

Todos os autores fizeram contribuições substanciais para concepção, ou delineamento, ou aquisição, ou análise ou interpretação de dados; e redação do trabalho ou revisão crítica; e aprovação final da versão para publicação.

\section{Disponibilidade dos dados e responsabilidade pelos resultados}

Todos os autores declaram ter tido total acesso aos dados obtidos e assumem completa responsabilidade pela integridade destes resultados.

\section{Referências}

1. Brasil. Ministério da Saúde. Portaria n. ${ }^{\circ}$ 1.727, de 11 de julho de 2017. Aprova o Plano Nacional de Assistência à Criança com Cardiopatia Congênita. Diário Oficial da República Federativa do Brasil [Online] 2017 maio [acesso 15 mar. 2020]. Disponivel em: https://portalarquivos2.saude.gov.br/images/pdf/2017/julho/31/Portaria-1727.pdf

2. Barbero-Marcial M, Tanamati C, Jatene MB, Aiello VD, Baucia JA, Atik E, et al. Dupla via de saída do ventrículo direito com comunicação interventricular não relacionada: resultados da correção cirúrgica com técnica de múltiplos retalhos. Rev Bras Cir Cardiovasc. 1997;12(2):160-65.

\section{http://dx.doi.org/10.1590/S0102-76381997000200009}

3. Hoeper MM, Bogaard HJ, Condliffe R, Frantz R, Khanna $D$, Kurzyna M, et al. Definitions and diagnosis of pulmonary hypertension. J Am Coll Cardiol. 2013;62(25):D42-50. https://doi.org/10.1016/j.jacc.2013.10.032

4. Kabitz HJ, Bremer HC, Schwoerer A, Sonntag F, Walterspacher S, Walker DJ, et al. The Combination of Exercise and Respiratory Training Improves Respiratory Muscle Function in Pulmonary Hypertension. Lung. 2014;192(2):321-28. https://doi: 10.1007/s00408-013-9542-9

5. Costa $\mathrm{CH}$, Rufino R. Hipertensão arterial pulmonar associada às cardiopatias congênitas. Pulmão RJ. 2015;24(2):43-6.
6. Enright PL, Sherrill DL. Reference equations for the six-minute walk in healthy adults. Am J Respir Crit Care Med. 1998; 158(5 Pt 1):1384-7. http://doi: 10.1164/ ajrccm.158.5.9710086

7. Neder JA, Andreoni S, Lerario MC, Nery LE. Reference values for lung function tests. II. Maximal respiratory pressures and voluntary ventilation. Braz J Med Biol Res. 1999:32(6):719-27. http://doi.org/10.1590/S0100879X1999000600007

8. Chia KSM, Faux SG, Wong PKK, Holloway C, Assareh $\mathrm{H}$, McLachlan CS, et al. Randomised controlled trial examining the effect of an outpatient exercise training programme on haemodynamics and cardiac MR parameters of right ventricular function in patients with pulmonary arterial hypertension: the ExPAH study protocol. BMJ Open. 2017:7(2):e014037. https://doi:10.1136/ bmjopen-2016-014037

9. Galiè N, Humbert M, Vachiery JL, Gibbs S, Lang I, Torbicki A, et al. ESC Scientific Document Group. 2015 ESC/ERS Guidelines for the diagnosis and treatment of pulmonary hypertension: The Joint Task Force for the Diagnosis and Treatment of Pulmonary Hypertension of the European Society of Cardiology (ESC) and the European Respiratory Society (ERS): Endorsed by: Association for European Paediatric and Congenital Cardiology (AEPC), International Society for Heart and Lung Transplantation (ISHLT). Eur Heart J. 2016;37(1):67119. https://doi: 10.1093/eurheartj/ehv317

10. Richter MJ, Grimminger J, Krüger B, Ghofrani HA, Mooren FC, Gall H, et al. Effects of exercise training on pulmonary hemodynamics, functional capacity and inflammation in pulmonary hypertension. Pulm Circ. 2017:7(1): 20-37. https://doi:10.1086/690553

11. Saglam M, Vardar-Yagli N, Calik-Kutukcu E, Arikan H, Savci S, Inal-Ince D, et al. Functional exercise capacity, physical activity, and respiratory and peripheral muscle strength in pulmonary hypertension according severity. J Phys Ther Sci. 2015;27(5):1309-12. https://doi:10.1589/jpts.27.1309

12. Pandey A, Garg S, Khunger M, Garg S, Kumbhani DJ, Chin KM, et al. Efficacy and safety of exercise training in chronic pulmonar hyoertencion: systematic review and meta-analysis. Circ heart fail. 2015;8(6):1032-43. https:// doi.org/10.1161/circheartfailure.115.002130

\section{Andrieli Barbieri Garlet}

Especialista em Fisioterapia Hospitalar com ênfase em Cardiologia pelo Instituto de Cardiologia do Rio Grande do Sul (ICFUC/RS), em Porto Alegre, RS, Brasil; Fisioterapeuta pela Universidade Federal de Santa Maria (UFSM), em Santa Maria, RS, Brasil.

\section{Aline Tibola}

Especialista em Fisioterapia Hospitalar com ênfase em Cardiologia pelo Instituto de Cardiologia do Rio Grande do Sul (ICFUC/RS), em Porto Alegre, RS, Brasil; Fisioterapeuta pela Universidade de Passo Fundo (UPF), em Passo Fundo, RS, Brasil. 


\section{Bruna Eibel}

Doutora em Ciências da Saúde: Cardiologia, pelo Instituto de Cardiologia do Rio Grande do Sul (ICFUC/ RS), em Porto Alegre, RS, Brasil; Coordenadora e preceptora do Programa de Residência Multiprofissional Integrada em Saúde: Cardiologia/Fisioterapia Instituto de Cardiologia do Rio Grande do Sul (ICFUC/RS), em Porto Alegre, RS, Brasil.

\section{Endereço para correspondência}

Bruna Eibel

Fundação Universitária de Cardiologia

Av. Princesa Isabel, 370

Santana, 90620-001

Porto Alegre, RS, Brasil 